\title{
Correction to: Transposable Elements Activity is Positively Related to Rate of Speciation in Mammals
}

\author{
Marco Ricci $^{1}(\mathbb{D}) \cdot$ Valentina Peona $^{1,2} \mathbb{D} \cdot$ Etienne Guichard $^{1}\left(\mathbb{D} \cdot\right.$ Cristian Taccioli $^{3} \mathbb{D} \cdot$ Alessio Boattini $^{1}$ (D)
}

Published online: 25 June 2018

(c) The Author(s) 2018

\section{Correction to: Journal of Molecular Evolution (2018) https://doi.org/10.1007/s00239-018-9847-7}

The original version of the article unfortunately contained tagging error in Given and Surname of all the authors. This has been corrected with this erratum.

Marco Ricci-Given name: Marco; Surname: Ricci

Valentina Peona-Given name: Valentina; Surname: Peona
Etienne Guichard-Given name: Etienne; Surname: Guichard

Cristian Taccioli-Given name: Cristian; Surname: Taccioli

Alessio Boattini-Given name: Alessio; Surname: Boattini

The original article has been corrected.

Marco Ricci, Valentina Peona as well as Cristian Taccioli, Alessio Boattini have contributed equally to this study.

The original article can be found online at https://doi.org/10.1007/ s00239-018-9847-7.

Marco Ricci

marco.ricci19@unibo.it

$\square$ Etienne Guichard

etienne.guichard2@unibo.it

1 Department of Biological, Geological and Environmental Sciences, University of Bologna, Bologna, Italy

2 Department of Ecology and Genetics, University of Uppsala, Uppsala, Sweden

3 Department of Animal Medicine, Health and Production, University of Padova, Padova, Italy 